Relations industrielles

Industrial Relations

\title{
Amendements au Code du travail du Québec (Bill 50)
}

\section{Pierre Verge}

Volume 24, numéro 3, 1969

URI : https://id.erudit.org/iderudit/028049ar

DOI : https://doi.org/10.7202/028049ar

Aller au sommaire du numéro

\section{Éditeur(s)}

Département des relations industrielles de l'Université Laval

\section{ISSN}

0034-379X (imprimé)

1703-8138 (numérique)

Découvrir la revue

\section{Citer cet article}

Verge, P. (1969). Amendements au Code du travail du Québec (Bill 50). Relations industrielles / Industrial Relations, 24(3), 603-622.

https://doi.org/10.7202/028049ar

Tous droits réservés @ Département des relations industrielles de l'Université Laval, 1969
Ce document est protégé par la loi sur le droit d'auteur. L'utilisation des services d’Érudit (y compris la reproduction) est assujettie à sa politique d'utilisation que vous pouvez consulter en ligne.

https://apropos.erudit.org/fr/usagers/politique-dutilisation/ 


\section{INFORMATIONS}

\section{Amendements au Code du travail du Québec (Bill 50)}

\section{Pierre Verge}

La loi modifiant le Code du travail, Bill 50 (1969), sanctionnée le 13 juin 1969, apporte essentiellement les changements majeurs suivants au Code du travail, S.R.Q., 1964, c. 141 :

- remplacement de la Commission des relations du travail du Québec par des fonctionnaires, soit des enquêteurs et des commissairesenquêteurs;

- création d'un « Tribunal du travail », composé de juges de la Cour provinciale et ayant notamment juridiction pour décider en appel de toute décision d'un commissaire-enquêteur et, en première instance, de toute poursuite pénale intentée en vertu du Code du travail;

- suppression du régime de la reconnaissance volontaire des associations de salariés par l'employeur;

- énoncé du droit d'un intéressé (ce qui comprendrait vraisemblablement un salarié agissant indépendamment de l'association accréditée) d'obtenir l'arbitrage d'un grief.

À noter que cette loi «entrera en vigueur à la date ou aux dates fixées par une ou plusieurs proclamations du lieutenant-gouverneur en conseil (Bill 50, a. 49).

Voici le texte du Code du travail compte tenu de ces nouveaux amendements, lesquels y figurent entre parenthèses.

* Les amendements apportés au Code du travail du Québec par le Bill 50 sont reproduits en caractères italiques et les références à cette loi modifiant le Code du travail sont inscrites à la suite de chacun des articles amendés. 


\section{TITRE PREMIER}

DES RELATIONS $d u$ TRAVAIL

(Am. Bill 50, a. 1.)

\section{Chapitre I}

\section{DÉFINITIONS}

1. Dans le présent code, à moins que le contexte ne s'y oppose, les termes suivants signifient :

a) «association de salariés $\gg$ - un groupement de salariés constitué en syndicat professionnel, union, fraternité ou autrement et ayant pour buts l'étude, la sauvegarde et le développement des intérêts économiques, sociaux et éducatifs de ses membres et particulièrement la négociation et l'application de conventions collectives;

b) «association accréditée 》-l'association reconnue par décision de l'enquêteur, du commissaire-enquêteur ou du tribunal comme représentant de l'ersemble ou d'un groupe des salariés d'un employeur;

d) «association d'employeurs - un groupement d'employeurs ayant pour buts l'étude et la sauvegarde des intérêts économiques de ses membres et particulièrement l'assistance dans la négociation et l'application de conventions collectives;

e) «convention collective»-une entente écrite relative aux conditions de travail conclue entre une ou plusieurs associations accréditées et un ou plusieurs employeurs ou associations d'employeurs;

f) «différend»-une mésentente relative à la négociation ou au renouvellement d'une convention collective ou à sa revision par les parties en vertu d'une clause la permettant expressément ;

$g$ ) 《grief - toute mésentente relative à l'interprétation ou à l'application d'une convention collective ;

h) « grève - la cessation concertée de travail par un groupe de salariés ;

i) «lock out» - le refus par un employeur de fournir du travail à un groupe de salariés à son emploi en vue de les contraindre à accepter certaines conditions de travail ou de contraindre pareillement des salariés d'un autre employeur;

j) «tribunal - le tribunal institué par la présente loi;

k) «ministre - le ministre du travail et de la main-d'oeuvre;

l) «employeur - quiconque, y compris Sa Majesté, fait exécuter un travail par un salarié ;

$m$ ) «salarié \$- une personne qui travaille pour un employeur moyennant rémunération, cependant ce mot ne comprend pas :

$1^{\circ}$ une personne qui, au jugement du commissaire-enquêteur, est employée à titre de gérant, surintendant, contremaître ou représentant de l'employeur dans ses relations avec ses salariés;

$2^{\circ}$ un administrateur ou officier d'une corporation;

$3^{\circ}$ un fonctionnaire du gouvernement dont l'emploi est, au jugement d'un commissaire-enquêteur, d'un caractère confidentiel, tel que celui d'un conciliateur du ministère du travail et de la main-d'oeuvre, d'un enquêteur visé par la présente 
loi, d'un employé du Conseil exécutif, du Conseil de la trésorerie, de la Commission de la fonction publique ou du cabinet d'un ministre, ou d'un directeur du personnel;

$4^{\circ}$ un membre de la Sûreté provinciale du Québec;

n) «services publics》— les catégories suivantes d'employeurs :

$1^{\circ}$ les corporations municipales et scolaires;

$2^{\circ}$ les hôpitaux, les sanatoriums et les institutions pour malades mentaux;

$3^{\circ}$ les hospices, les crèches et les orphelinats ;

$4^{\circ}$ les universités, les collèges et les couvents ;

$5^{\circ}$ les entreprises de téléphone, de télégraphe, de transport par bateaux, tramways, autobus ou chemins de fer ;

$6^{\circ}$ les entreprises de production, transport, distribution ou vente de gaz, d'eau ou d'électricité et les services de transport par véhicules de livraison munis d'une autorisation de la Régie des transports;

$7^{\circ}$ les entreprises d'enlèvement d'ordures ménagères ;

$8^{\circ}$ les services du gouvernement de la province et les autres agents de Sa Majesté du chef de la province, à l'exception de la Régie des alcools du Québec ;

o) «exploitation forestière »- la coupe, le tronçonnement, l'écorçage en forêt, le charroyage, l'empilement, le flottage, le chargement et le transport routier du bois à l'exclusion de sa transformation en dehors de la forêt ;

p) «concessionnaire forestier»-le détenteur du droit de coupe de bois ou le propriétaire du fond ou le détenteur du permis de coupe lorsqu'il n'a pas cédé le droit de coupe à un tiers.

(Am. Bill 50 a. 2.)

2. Le concessionnaire forestier est, pour les fins des chapitres II et III, réputé employeur de tous salariés employés à l'exploitation forestière de ses terres sauf ceux qui sont employés au transport routier.

Le commissaire-enquêteur en chef peut cependant reconnaître une association d'employeurs comme représentant de tous les employeurs faisant l'exploitation forestière des terres d'un concessionnaire forestier ou d'une partie déterminée de ces terres; cette association est alors réputée employeur de la façon ci-dessus indiquée.

Le présent article ne s'applique pas aux salariés membres d'une coopérative faisant des travaux d'exploitation forestière. (Am. Bill 50, a. 3.)

\section{Chapitre II}

\section{DES ASSOCIATIONS}

\section{Section I}

DU DROIT D'ASSOCIATION

3. Tout salarié a droit d'appartenir à une association de salariés de son choix, et de participer à ses activités et à son administration. 
4. Les policiers municipaaux ne peuvent être membres d'une association de salariés qui n'est pas formée exclusivement de policiers municipaux ou qui est affiliée à une autre organisation.

5. Personne ne peut, au nom ou pour le compte d'une association de salariés, solliciter, pendant les heures de travail, l'adhésion d'un salarié à une association.

6. Une association de salariés ne doit tenir aucune réunion de ses membres au lieu du travail sauf si elle est accréditée et du consentement de l'employeur.

7. Dans une exploitation forestière, les lieux affectés aux repas des salariés ne sont pas considérés comme lieux de travail et aucune réunion ne peut être tenue dans les lieux affectés au logement des salariés.

8. Sous réserve de la Loi des terres et forêts, le propriétaire d'une terre ou concession où se fait une exploitation forestière est tenu de permettre le passage et de donner accès au campement des salariés à tout représentant d'une association de salarié muni d'un permis délivré par le commissaire-enquêteur en chef.

L'exploitant est tenu de fournir à ce représentant le gîte et le couvert au prix fixé pour les salariés par ordonnance suivant la Loi dı salaire minimum.

Il doit sur demande écrite d'un salarié lui avancer la somme requise pour droit d'entrée dans une association et première cotisation pourvu que ce salarié ait cette somme à son crédit.

Le présent article ne s'applique pas à l'exploitation forestière effectuée sur sa propriété par un cultivateur ou colon. (Am. Bill 50, a. 4.)

9. Sous réserve de la Loi des terres et forêts, le propriétaire d'une entreprise minière où des salariés sont logés sur des terrains auxquels il est en mesure d'interdire l'accès doit accorder cet accès à tout représentant d'une association de salariés muni d'un permis délivré par le ministre.

L'exploitant d'une telle entreprise est tenu de fournir à ce représentant le gîte et le couvert au prix courant pour les salariés. (Am. Bill 50, a. 5.)

10. Tout employeur a droit d'appartenir à l'association d'employeurs de son choix et de participer à ses activités et à son administration.

10a. Une corporation scolaire peut donner à une commission scolaire régionale ou à une association de corporations scolaires un mandat exclusif pour les fins des articles 40 à 81 .

Ce mandat n'est révocable qu'au temps fixé par l'article 21 pour une demande d'accréditation.

Il appartient à un commissaire-enquêteur de statuer sur la validité de ce mandat.

Tant qu'il est en vigueur, les obligations prévues aux articles 41 et 44 incombent exclusivement au mandataire; cependant celui-ci ne peut contraindre une association de salariés à négocier une convention collective qui s'applique à un territoire excédant celui d'une commission scolaire régionale. (13-14 Elizabeth II, 1965, chap. 50, a. 1) (Am. Bill 50, a. 6.) 
11. Aucun employeur, ni aucune personne agissant pour un employeur ou une association d'employeurs, ne cherchera d'aucune manière à dominer, entraver ou financer la formation ou les activités d'une association de salariés, ni à y participer.

Aucune association de salariés, ni aucune personne agissant pour le compte d'une telle organisation n'adhèrera à une association d'employeurs, ni ne cherchera à dominer, entraver ou financer la formation ou les activités d'une telle association ni à y participer.

12. Nul ne doit user d'intimidation ou de menaces pour amener quiconque à devenir membre, à s'abstenir de devenir membre ou à cesser d'être membre d'une association de salariés ou d'employeurs, ni pour amener un salarié à signer, à refuser, à révoquer ou à rétablir une autorisation de retenir un montant sur son salaire comme cotisation.

13. Aucun employeur, ni aucune personne agissant pour un employeur ou une association d'employeurs ne doit refuser d'employer une personne parce qu'elle est membre ou officier d'une association, ni chercher par intimidation, menace de renvoi ou autre menace, ou par l'imposition d'une peine ou par quelque autre moyen, à contraindre un salarié à s'abstenir de devenir membre ou officier ou à cesser d'être membre ou officier d'une association de salariés.

Le présent article n'a pas pour effet d'empêcher un employeur de suspendre, congédier ou déplacer un salarié pour une cause juste et suffisante dont la preuve lui incombe.

14. Lorsqu'un salarié est congédié, suspendu ou déplacé par l'employeur ou son agent à cause de l'exercice par ce salarié d'un droit qui lui résulte du présent code, le commissaire-enquêteur peut ordonner à l'employeur de réintégrer, dans les huit jours de la signification de la décision, ce salarié dans son emploi avec tous ses droits et privilèges, et de lui payer, à titre d'indemnité, l'équivalent du salaire et des autres avantages dont l'a privé le congédiement, la suspension ou le déplacement.

Si le salarié a travaillé ailleurs au cours de la période précitée, le salaire qu'il a ainsi gagné doit être déduit de cette indemnité. (Am. Bill 50, a. 7.)

15. Le salarié qui croit avoir été illégalement congédié, suspendu ou déplacé pour une cause mentionnée à l'article précédent doit, s'il désire se prévaloir des dispositions de cet article, soumettre sa plainte par écrit au commissaire-enquêteur dans les quinze jours du congédiement, de la suspension ou du déplacement ou la mettre à la poste à l'adresse du commissaire-enquêteur dans ce délai.

(Am. Bill 50, a. 7.)

16. S'il est établi à la satisfaction $d u$ commissaire-enquêteur que le salarié exerce un droit lui résultant du présent code, il y a présomption en sa faveur qu'il a été congédié, suspendu ou déplacé à cause de l'exercice de ce droit, et il incombe à l'employeur de prouver que le salarié a été congédié, suspendu ou déplacé pour une autre cause, juste et suffisante.

(Am. Bill 50, a.7.)

17. Le salarié qui refuse de reprendre son emploi, quoique dûment rappelé au travail par l'employeur, n'a droit à l'indemnité que jusqu'au jour où il a été rappelé au travail. 
18. Au cas de contestation entre l'employeur et le salarié quant au montant d'une indemnité, le quantum en est fixé par un commissaire-enquêteur.

Celui-ci peut exercer, pour le compte du salarié, le recours qui résulte de sa décision, à défaut du salarié de le faire dans les vingt-quatre jours.

L'action en recouvrement de l'indemnité par le salarié ou le commissaireenquêteur est intentée devant le tribunal de iuridiction civile compétent en raison du montant réclamé.

Ce recours se prescrit par six mois à compter de la décision du commissaireenquêteur fixant le quantum. (Rempl. Bill 50, a. 8.)

19. Les dispositions de l'article 118 s'appliquent, mutatis mutandis, aux décisions visées par les articles ci-dessus.

\section{SECTION II}

DE L'ACCRÉDITATION

20. A droit à l'accréditation l'association de salariés groupant la majorite absolue des salariés d'un employeur.

Ce droit existe à l'égard de la totalité des salariés de l'employeur ou de chaque groupe desdits salariés que l'enquêteur ou le commissaire-enquêteur déclare devoir former un groupe distinct aux fins du présent code.

Les salariés membres de chacune des professions visées aux chapitres 247 à 249, 253 à 255 et 257 à 266 des Statuts refondus, 1964, forment obligatoirement avec les personnes admises à l'étude de chaque profession un groupe distinct.

Un seul salarié peut former un groupe aux fins du présent article.

Les personnes employées à l'exploitation d'une ferme ne sont pas réputées ćtre des salariés aux fins de la présente section, à moins qu'elle n'y soient ordinairement et continuellement employées au nombre minimal de trois.

(Rempl. Bill 50, a.9.)

21. L'accréditation peut être demandée:

a) en tout temps, à l'égard d'un groupe de salariés qui n'est pas représenté par une association accréditée ;

b) après dix mois de la date d'une accréditation, à l'égard d'un groupe de salariés pour lesquels une convention collective n'a pas été conclue ou un différend soumis à l'arbitrage;

c) après six mois de l'expiration des délais prévus à l'article 46, à l'égard d'un groupe de salariés pour lesquels une convention collective n'a pas été conclue et le différend ne fait pas l'objet d'une grève ou d'un lock-out permis par le présent code;

d) du soixantième au trentième jour précédant la date d'expiration d'une convention collective ou de son renouvellement ou l'expiration d'une sentence arbitrale en tenant lieu.

21a. Le lieutenant-gouverneur en conseil nomme au ministère du iravail et de la main-d'oeuvre, en conformité de la Loi de la fonction publique, un commissaire-enquêteur en chef, des commissaires-enquêteurs, des enquêteurs et le personnet 
nécessaire pour assurer l'efficacité de la procédure d'accréditation établie par la présente loi.

En outre de ses pouvoirs et devoirs comme commissaire-enquêteur et des attributions particulières qui lui sont assignées par les dispositions qui suivent, le le commissaire-enquêteur en chef dirige, coordonne et distribue le travail des commissaires-enquêteurs et des enquêteurs et généralement veille au bon fonctionnemenı de ce service. (Aj. Bill 50, a.10)

22. L'accréditation est demandée au ministre par une association de salariés, au moyen d'une requête autorisée par résolution, signée par ses représentants mandatés et indiquant le groupe qu'elle veut représenter. L'association doit transmettre une copie de cette requête à l'employeur, lequel, dans les cinq jours de sa réception, doit afficher, dans un endroit bien en vue, la liste complète des salariés de l'entreprise visés par la requête. L'employeur doit transmettre sans délai une copie de cette liste à l'association requérante. (Rempl. Bill 50, a.11.)

23. La requête doit être accompagnée d'une copie certifiée de la constitution et des règlements de l'association ainsi que d'un état des conditions d'admission, droits d'entrée et cotisations exigés de ses membres.

24. Dès réception de la requête, le ministre en affiche une copie au greffe du ministère à Montréal ou à Québec selon le cas. Ce greffe est accessible au public.

De plus, le ministre dépêche sans délai un enquêteur qui doit s'assurer du caractère représentatif de l'association et de son droit à l'accréditation. À cette fin, l'enquêteur procède à la vérification des livres et archives de l'association et de la liste des salariés de l'employeur. S'il vient à la conclusion que l'association jouit du caractère représentatif et s'il constate qu'il y a accord entre l'employeur et l'association sur l'unité de négociations et sur les personnes qu'elle vise, il doit l'accréditer sur-le-champ et par écrit (Rempl. Bill 50, a.12.)

24a. Si l'enquêteur est d'avis que l'association n'est pas représentative ou s'il n'y a pas accord entre l'employeur et l'association sur l'unité de négociation, il doit faire un rapport sommaire de son enquête au commissaire-enquêteur en chef et en transmettre une copie à l'association et à l'employeur. Ce rapport doit indiquer les raisons pour lesquelles il a refusé l'accréditation. (Rempl. Bill 50, a.12.)

24b. Le commissaire-enquêteur est investi de tous les pouvoirs, immunités et privilèges d'un commissaire nommé en vertu de la Loi des commissions d'enquête; il a en particulier le pouvoir d'assigner des témoins, d'exiger et de recevoir la prestation du serment, d'obliger toute personne à déposer des pièces ou documents en sa possession et généralement de poser tout acte nécessaire à la poursuite de l'objet de son enquête.

Le commissaire-enquêteur doit décider, après enquête sur les lieux auprès de l'association requérante, de son caractère représentatif. Il doit aussi trancher, après enquête tenue en présence de l'association et de l'employeur, tout différend relatif à l'unité de négociation et aux personnes qu'elle vise.

Sitôt son enquête terminée ou au plus tard dans les trois jours suivants, le commissaire-enquêteur doit rendre sa décision à l'effet d'accorder ou de refuser l'accréditation et, le cas échéant, décrire l'unité de négociation appropriée. Cette décision doit être rendue par écrit et motivée. Des copies certifiées conformes doivent en être transmises à l'association et à l'employeur. 
La transcription des notes sténographiques de l'enquête ou de son enregistrement sur bande magnétique, les pièces ou documents déposés et la décision du commissaire-enquêteur constituent le dossier de l'enquête. (Rempl. Bill 50, a.12.)

24c. Dans tout acte de procédure, le nom de tout adhérent à une association ne doit être révélé qu'à l'enquêteur, au commissaire-enquêteur, au commissaire- enquêteur en chef ou au tribunal. Ces personnes sont tenues au secret.

(Rempl. Bill 50, a.12.)

25. Le commissaire-enquêteur peut ordonner le vote au scrutin secret d'un groupe désigné de salariés chaque fois qu'il le juge opportun et, en particulier, lorsqu'il est d'avis qu'une contrainte a été exercée pour empêcher un certain nombre des dits salariés d'adhérer à une association de salariés ou pour les forcer à y adhérer, ou s'il appert que les dits salariés sont membres de plus d'une association en nombre suffisant pour influer sur la décision. (Am. Bill 50, a.13.)

26. Tout employeur est tenu de faciliter la tenue du scrutin et tout salarié faisant partie d'un groupe désigné par le commissaire-enquêteur est tenu de voter à moins d'une excuse légitime. (Am. Bill 50, a.13)

27. (Abrogé, Bill 50, a.14)

28. Toute plainte portée par un tiers au commissaire-enquêteur sur une violation de l'article 11 suspend l'enquête de l'enquêteur. Sur réception de la plainte, le commissaire-enquêteur en vérifie le bien-fondé et accorde ou refuse la demande d'accréditation suivant la procédure prévue à l'article 24b. (Rempl. Bill 50, a.15.)

Une copie de cette décision doit être transmise à l'employeur.

29. (Abrogé, Bill 50, a.16)

30. De plein droit, au cours de son enquête, et en tout temps sur requête d'une partie intéressée, le commissaire-enquêteur peut décider si une personne est un salarié ou un membre d'une association, si elle est comprise dans l'unité de négociation, et toutes autres questions relatives à l'accréditation. (Am. Bill 50, a.17.)

31. Une requête en accréditation ne peut être renouvelée avant trois mois de de son rejet par un commissaire-enquêteur. (Am. Bill 50, a.18.)

32. Au temps fixé aux paragraphes $b, c$ et $d$ de l'article 21 , un commissaire. enquêteur peut révoquer l'accréditation pour les causes suivantes:

a) si l'association a cessé d'exister;

b) si elle ne représente plus la majorité du groupe pour lequel elle est accréditée. (Am. Bill 50, a.19.)

33. Lorsqu'il est saisi d'une requête en accréditation, révision ou révocation d'accréditation, un commissaire-enquêteur peut ordonner la suspension des négociations et des délais de négociations collectives et empêcher le renouvellement d'une convention collective.

En ce cas, les conditions de travail prévues dans cette convention demeurent en vigueur jusqu'à la décision du commissaire-enquêteur et les dispositions de l'article 48 s'appliquent. (Bill 50, a.20.) 
34. L'accréditation d'une association de salariés annule de plein droit l'accréditation de toute autre association pour le groupe visé par la nouvelle accréditation. (Am. Bill 50, a.21.)

35. La révocation de l'accréditation empêche le renouvellement de toute convention collective conclue par l'association privée de son accréditation et emporte aussi de plein droit pour cette dernière la déchéance des droits et avantages lui résultant de cette convention collective. (Am. Bill 50, a.22.)

36. L'aliénation ou la concession totale ou partielle d'une entreprise autrement que par vente en justice n'invalide aucun certificat délivré par un commissaireenquêteur, aucune convention collective, ni aucune procédure en vue de l'obtention d'un certificat ou de la conclusion ou de l'exécution d'une convention collective.

Sans égard à la division, à la fusion ou au changement de structure juridique de l'entreprise, le nouvel employeur est lié par le certificat ou la convention collective comme s'il y était nommé et devient par le fait même partie à toute procédure s'y rapportant, aux lieu et place de l'employeur précédent.

(Am. Bill 50 a.23.)

37. Le Commissaire-enquêteur en chef peut rendre toute ordonnance jugée nécessaire pour constater la transmission de droits et d'obligations visée à l'article 36 et régler toute difficulté découlant de l'application du dit article (Am. Bill 50, a. 24.)

38. Un employeur est tenu d'honorer l'autorisation écrite volontaire et révocable donnée par tout salarié, membre d'une association accréditée, de retenir mensuellement un montant spécifié comme cotisation à prélever sur son salaire au bénéfice de cette dernière.

L'employeur est tenu de remettre mensuellement à l'association accréditée les montants ainsi retenus avec un état indiquant le montant prélevé de chaque salarié et le nom de celui-ci.

S'il reçoit une révocation, il doit en remettre une copie à l'association.

39. Une association accréditée doit informer annuellement le commissaireenquêteur en chef, à l'époque et dans la forme que celle-ci détermine, de tout changement de ses constitution et règlements ainsi que des noms et adresses de ses officiers (Am. Bill 50, a.25.)

39a. Un commissaire-enquêteur peut, pour cause, réviser ou révoquer toute décision et tout ordre qu'il a rendu et tout certificat qu'il a délivré. (Aj. Bill 50, a.26.)

39b. Le commissaire-enquêteur doit avant de rendre une décision sur la révocation ou la révision pour cause d'une décision ou d'un ordre qu'il a rendu. permettre aux parties de se faire entendre et, à cette fin, leur donner, en la manière qu'il juge appropriée, un avis d'au moins cinq jours francs de la date, de l'heure et du lieu où elles pourront se faire entendre.

Si une partie intéressée et ainsi convoquée ne se présente pas ou refuse de se faire entendre d̀ la séance fixée pour cette fin, ou à un ajournement de cette séance, le commissaire-enquêteur peut néanmoins procéder à l'instruction de l'affaire et aucun recours judiciaire ne peut être fondé sur le fait qu'il a ainsi procédé en l'absence de cette partie. (Aj. Bill 50, a.26.) 
39c. Une copie de toute décision d'un enquêteur ou d'un commissaire-enquêteur accordant une accréditation doit être transmise sans délai au ministre.

(Aj. Bill 50, a.26.)

\section{Chapitre III}

40. L'association accréditée donne à l'employeur, ou celui-ci donne d̀ l'association accréditée, un avis écrit d'au moins huit jours de la date, de l'heure et du lieu où ses représentants seront prêts à rencontrer l'autre partie ou ses représentants pour la conclusion d'une convention collective.

Une partie à une convention collective peut donner un semblable avis dans les soixante jours précédant son expiration à moins qu'un autre délai n'y soit fixé.

L'association accréditée et l'employeur peuvent donner un semblable avis dans les soixante jours précédant l'expiration d'une sentence arbitrale tenant lieu de convention collective.

L'association accréditée et l'employeur ne doivent conclure qu'une seule convention collective à l'égard d'un groupe distinct décrété par un enquêteur ou un commissaire-enquêteur. (Rempl. Bill 50,a.27.)

41. Après un avis prévu à l'article précédent, les négociations doivent commencer et se poursuivre avec diligence et bonne foi.

42. Si les négociations se sont poursuivies sans succès pendant trente jours ou si l'une des parties ne croit pas qu'elles puissent être complétées dans un délai raisonnable, chaque partie peut en donner avis au ministre en lui exposant les difficultés rencontrées.

Cet avis doit être en même temps notifié à l'autre partie.

43. Sur réception de cet avis, le ministre charge un conciliateur de rencontrer les parties et de tenter d'effectuer une entente.

44. Les parties au différend ont l'obligation d'assister à toutes réunions où le conciliateur les convoque.

45. Le conciliateur fait rapport au ministre dans les trente jours de sa nomination ou dans le délai ultérieur dont les parties conviennent par écrit.

46. Si l'intervention du conciliateur a été infructueuse, le droit à la grève ou au lock-out est acquis soixante jours ou, s'il s'agit de la conclusion d'une première convention, quatre-vingt-dix jours, après la réception par le ministre de l'avis prévu à l'article 43, à moins que les parties ne soumettent leur différend à un conseil d'arbitrage.

47. À compter du dépôt d'une requête en accréditation d'une association et tant que le droit au lock-out n'est pas acquis, un employeur ne doit pas, sans le consentement écrit de l'association requérante ou une sentence arbitrale, modifier les conditions de travail de ses salariés et il est tenu, s'il est alors lié par une convention collective, de continuer à s'y conformer. (Am. Bill 50, a.28.)

48. Pendant la période visée à l'article 47 , il est interdit de conseiller ou d'enjoindre à des salariés de ne pas continuer à fournir leurs services à leur employeur aux mêmes conditions de travail. 
49. Une association accréditée est subrogée de plein droit dans tous les droits et obligations résultant d'une convention collective en vigueur conclue par une autre association; cependant elle peut y mettre fin ou la déclarer non avenue par avis écrit transmis à l'employeur et au commissaire-enquêteur en chef.

(Am. Bill 50, a.29.)

50. La convention collective peut contenir toute disposition relative aux conditions de travail qui n'est pas contraire à l'ordre public ni prohibée par la loi.

Elle ne doit contenir aucune clause ou condition venant en conflit avec les droits et pouvoirs attribués par la loi aux autorités municipales ou scolaires en matière d'engagement, de suspension et de renvoi de leurs employés.

51. Chaque partie peut exiger que la convention collective soit rédigée dans les deux langues française et anglaise.

52. Une convention collective n'est pas invalidée par la nullitée d'une ou plusieurs de ses clauses.

53. La durée d'une convention collective est d'au moins un an et d'au plus trois ans. (13-14 Elizabeth II, 1965, chap. 50, a. 3)

54. Est présumée en vigueur pour la durée d'une année, la convention ne comportant pas de terme fixe et certain.

55. Lo convention collective lie tous les salariés actuels ou futurs visés par l'accréditation. (Rempl. Bill 50, a.30.)

56. La convention collective conclue par une association d'employeurs lie tous les employeurs membres de cette association auxquels elle est susceptible de s'appliquer, y compris ceux qui y adhèrent ultérieurement.

La convention collective conclue par une association de corporations scolaires ne lie que celles qui lui ont donné le mandat exclusif prévue à l'article $10 a$. (13-14 Elizabeth II, 1965, chap. 50, a. 4)

57. L'association accréditée [...] peut exercer tous les recours que la convention collective accorde à chacun des salariés qu'elle représente sans avoir à justifier d'une cession de créance de l'intéressé. (Am. Bill 50, a.31.)

58. Les recours de plusieurs salariés contre un même employeur peuvent être cumulés dans une seule demande et le total réclamé détermine la compétence tant en première instance qu'en appel.

59. Les droits et recours qui naissent d'une convention collective ou d'une sentence qui en tient lieu se prescrivent par six mois à compter du jour où la cause de l'action a pris naissance. Le recours à la procédure de griefs interrompt la prescription.

60. Dix exemplaires ou copies certifiées conformes de toute convention collective, ainsi au'une copie certifiée conforme de toute annexe, doivent être déposés dans les soixante jours au bureau du commissaire-enquêteur en chef. Le défaut de dépôt prévu ci-dessus donne ouverture à une demande d'accréditation par une autre association. 
Une convention collectivve ne prend effet qu'à compter de son dépôt au bureau du commissaire-enquêteur en chef.

La partie qui fait ce dépôt doit indiquer le nombre de salariés régis par la convention collective. (Rempl. Bill 50, a.32.)

61. Nulle association accréditée ayant conclu une convention collective, nul groupe de salariés régis par une telle convention ou par une sentence arbitrale en ayant l'effet, ne fera de démarches en vue de s'y affilier, sauf dans les soixante jours précédant la date d'expiration ou de renouvellement de la convention ou la date d'expiration de la sentence arbitrale. (Am. Bill 50, a. 33.)

\section{Chapitre IV}

DU RÈGLEMENT DES DIFFÉRENDS

\section{SECTION .II}

\section{DES CONSEILS D'ARBITRAGE}

62. Un différend est soumis à un conseil d'arbitrage sur demande écrite adressée au ministre par les parties.

63. Un conseil d'arbitrage se compose de trois membres, citoyens canadiens, majeurs et nommés par le ministre.

64. Les membres d'un conseil d'arbitrage ne doivent avoir aucun intérêt pécuniaire dans le différend qui leur est soumis ni avoir agi dans ce différend à titre d'agent d'affaires, de procureur, de conseiller ou de représentant d'une partie.

65. Le ministre nomme membres du conseil d'arbitrage les personnes désignées par chaque partie dans la demande.

Il nomme également le greffier.

66. Dans les cinq jours de leur nomination, les deux membres du conseil doivent se consulter sur le choix d'un troisième membre qui agira comme président; s'ils s'entendent, le ministre nomme à ce poste la personne de leur choix. À défaut d'entente, le ministre le nomme d'office.

Tout président nommé d'office est choisi sur une liste d'au moins vingt-cinq noms dressée annuellement par le ministre après consultation du Conseil consultatif du travail et de la main d'oeuvre. (Am. Bill 50, a. 34.)

67. Tout membre d'un conseil doit avant d'agir prêter serment de rendre sentence selon l'équité et la bonne conscience.

68. Toute vacance créée par décès, démission, incapacité ou refus d'agir d'un membre du conseil est remplie par le ministre suivant la procédure établie pour la nomination originale.

Au défaut d'une partie de désigner dans les dix jours de la demande du ministre le remplaçant du membre qu'elle a désigné, le ministre le nomme d'office.

69. Le conseil procède en toute diligence à l'instruction du différend selon la procédure et le mode de preuve qu'il juge appropriés. 
70. Les séances d'un conseil d'arbitrage sont publiques; le conseil peut toutefois, de son chef ou à la demande de l'une des parties, ordonner le huis clos.

71. Le président a tous les pouvoirs d'un juge de la Cour supérieure pour la conduite des séances du conseil; il ne peut cependant imposer l'emprisonnement.

72. Sur demande des parties ou du conseil, les témoins sont assignés par ordre écrit, signé par le greffier. Celui-ci peut faire prêter serment.

73. Une personne dûment assignée devant un conseil d'arbitrage qui refuse de comparaître ou de témoigner peut y être contrainte et être condamnée suivant la Loi des convictions sommaires de Québec, comme si elle avait été assignée suivant cette loi.

74. Les témoins ont droit à la même taxe que les témoins en Cour supérieure. Cette taxe est payable par la partie qui les a assignés ou interrogés.

75. Le greffier peut communiquer ou autrement signifier tout ordre, document ou procédure émanant du conseil ou des parties en cause.

76. La sentence d'un conseil d'arbitrage doit être motivée et signée par les membres qui y concourent.

Tout membre dissident peut faire un rapport distinct.

À défaut d'unanimité ou de majorité, le rapport du président constitue la sentence du conseil.

77. Le président transmet l'original de la sentence au ministre et en expédie, en même temps, une copie à chaque partie.

78. La sentence du conseil doit être rendue dans les soixante jours de la nomination du président à moins qu'à la demande du président, le ministre, s'il le juge dans l'intérêt de la justice et des parties, n'accorde un délai supplémentaire n'excédant pas trente jours, lequel peut, aux mêmes conditions, être prolongé à nouveau par le ministre.

79. En tout temps avant sa sentence finale, un conseil d'arbitrage peut rendre toute décision intérimaire qu'il croit juste et utile.

80. La sentence d'un conseil d'arbitrage lie les parties pour une durée n'excédant pas deux ans.

81. La sentence a l'effet d'une convention collective signée par les parties.

Elle peut être exécutée sous l'autorité d'un tribunal compétent, sur poursuite intentée par une partie, laquelle n'est pas tenue de mettre en cause la personne pour le bénéfice de laquelle elle agit.

\section{SECTION II}

\section{DES POLICIERS ET POMPIERS}

82. Tout différend entre une corporation municipale et une association de salariés accréditée pour représenter ses policiers ou pompiers est obligatoirement déféré par le ministre à un conseil d'arbitrage sur le rapport du conciliateur. (Am. Bill 50, a. 35.) 
83. Chaque partie est tenue de désigner un membre du conseil clans les dix jours de la demande qui lui est faite par le ministre. À son défaut de ce faire, le ministre peut désigner d'office et nommer une personne pour agir comme membre du conseil.

84. Les articles 63 à 81 s'appliquent au conseil d'arbitrage constitué en vertu de la présente section.

85. S'il survient entre une corporation municipale et une association visée à l'article 82 une mésentente autre qu'un différend ou un grief, le ministre peut charger un conciliateur de rencontrer les parties et de tenter d'effectuer une entente.

86. Sur le rapport du conciliateur le ministre peut, nonobstant l'article 90 , déférer la mésentente à un conseil d'arbitrage comme s'il s'agissait d'un différend.

87. Aucune disposition d'une sentence arbitrale, comportant une augmentation de dépenses pour une corporation municipale, n'est exécutoire avant l'expiration de son année financière en cours lors du prononcé de la sentence et ne peut rétroagir au-delà de douze mois à compter de cette sentence.

\section{SECTION III}

\section{DE L'ARBITRE DES GRIEFS}

88. Tout grief non satisfait doit, à la demande de l'intéressé, être soumis à l'arbitrage en la manière prévue dans la convention collective, si elle y pourvoit: sinon, il est déféré à un arbitre choisi par les parties ou, à défaut d'accord, nommé par le ministre.

Si la partie contre laquelle un grief est soulevé refuse de donner suite à la procédure de règlement des griefs prévue dans la convention collective, le grief est déféré à un arbitre nommé par le ministre. (Rempl. Bill 50, a.36.)

89. La sentence arbitrale est finale et lie les parties.

Elle peut être exécutée suivant l'article 31.

90. Pendant la durée d'une convention collective, toute mésentente autre qu'un grief au sens de l'article 1 ne peut être réglée que de la façon prévue dans la convention et dans la mesure où elle $y$ pourvoit.

\section{SECTION IV}

DE LA RÉGLEMENTATION

91. Le lieutenant-gouverneur en conseil détermine, par règlement, la rémunération des membres des conseils d'arbitrage, du tribunal d'arbitrage et des arbitres.

Il peut aussi faire tout règlement jugé nécessaire pour donner effet aux disposition du chapitre IV.

92. Ces règlements n'entrent en vigueur qu'après publication dans la Gazette officielle de Québec. 


\section{Chapitre $V$}

DES GRÈVES ET LOCK-OUT

93. Toute grève est interdite en toute circonstance aux policiers et pompiers à l'emploi d'une corporation municipale.

94. La grève est interdite tant qu'une association des salariés en cause n'a pas été accréditée et n'y a pas acquis droit suivant l'article 46. (Am. Bill 50, a. 37.)

95. La grève est prohibée pendant la durée d'une convention collective, à moins que celle-ci ne renferme une clause en permettant la revision par les parties et que les conditions prescrites à l'article précédent n'aient été observées.

96. Nulle association de salariés ou personne agissant dans l'intérêt d'une telle association ou d'un groupe de salariés n'ordonnera, n'encouragera ou n'appuiera un ralentissement d'activités destiné à limiter la production.

97. Le lock-out est interdit sauf dans le cas où une association de salariés a acquis droit à la grève.

98. Personne ne cesse d'être un salarié pour l'unique raison qu'il a cessé de travailler par suite de grève ou lock-out.

Rien dans le présent code n'empêche une interruption de travail qui ne constitue pas une grève ou un lock-out.

99. La grève est interdite aux salariés à l'emploi d'un service public à moins que l'association des salariés en cause y ait acquis droit suivant l'article 46 et ait donné par écrit au ministre avis préalable d'au moins huit jours lui indiquant le moment où elle entend y recourir.

Si le lieutenant-gouverneur en conseil est d'avis que dans un service public une grève appréhendée ou en cours met en danger la santé ou la sécurité publique, il peut constituer à ce sujet une commission d'enquête qui est investie des pouvoirs d'un conseil d'arbitrage pour faire enquête et rapport sur le différend, sauf qu'elle ne peut rendre une décision, ni formuler de recommandations, mais seulement constater les faits en se conformant aux articles 69 à 78 .

Sur la requête du procureur général après la constitution d'une commission d'enquête, un juge de la Cour supérieure peut, s'il est d'avis que la grève met en péril la santé ou la sécurité publique, décerner toute injonction jugée appropriée pour empêcher cette grève ou y mettre fin.

Une injonction décernée en vertu du présent article doit prendre fin au plus tard vingt jours après l'expiration du délai de soixante jours accordé à la commission d'enquête pour la production de son rapport, lequel délai ne peut être prolongé.

Le présent article s'applique à une grève appréhendée ou en cours qui compromet l'éducation d'un groupe d'élèves comme à une grève qui met en danger ou en péril la santé ou la sécurité publique. (13-14 Elizabeth II, 1965, chap. 50, a. 5) 


\section{Chapitre VI}

DU TRIBUNAL DU TRAVAIL

100. Un tribunal chargé de la décision des litiges concernant le travail est créé par la présente loi, sous le nom de «Tribunal du travail», avec les juridictions spécifiées ci-dessous.

(Rempl. Bill 50, a. 38.)

101. Après consultation $d u$ Conseil général du Barreau du Québec et $d u$ Conseil consultatif du travail et de la main-d'oeuvre, le lieutenant-gouverneur en conseil nomme, parmi des personnes versées dans les relations du travail, les membres du tribunal, soit: un juge en chef, un juge en chef adjoint et des juges de la Cour provinciale en nombre suffisant pour expédier rapidement les affaires qui leur sont soumises.

(Rempl. Bill 50, a. 38.)

102. Le lieutenant-gouverneur en conseil nomme, en conformité des dispositions de la Loi de la fonction publique, le personnel qu'il juge nécessaire au bon fonctionnement de ce tribunal.

(Rempl. Bill 50, a. 38.)

103. Ce tribunal a juridiction pour connaître et décider en dernier ressort tout litige surgissant à l'occasion de l'interprétation ou de l'application des dispositions du présent code et notamment,

a) en appel, de toute décision rendue par un commissaire-enquêteur;

b) en première instance, de toute poursuite pénale intentée en vertu du présent code.

(Rempl. Bill 50, a. 38.)

104. Un juge est compétent pour instruire et décider, seul, toute affaire soumise au tribunal du travail.

(Rempl. Bill 50, a. 38.)

105. La cause est instruite au chef-lieu du district judiciaire où elle a pris naissance, sauf si les parties en conviennent autrement, ou si le juge en chef décide, pour des raisons d'intérêt public, qu'elle sera instruite ailleurs.

(Rempl. Bill 50, a. 38.)

106. Peuvent en appeler d'une décision d'un commissaire-enquêteur:

a) en matière de refus ou d'octroi d'une accréditation, tout salarié compris dans l'unité de négociation ou l'association requérante ou une association qui étcit antérireurement accréditée;

b) en matière de description d'unité de négociation, ou d'inclusion ou d'exclusion des personnes qu'elle vise, l'employeur, l'association accréditée ou une association requérante rivale;

c) en matière de plainte pour violation de l'article 11, les tiers ou tout salarié compris dans l'unité de négociation ou une association requérante rivale.

* Les articles 100 à 120 du Code du travail ont été remplacés par les articles 100 à 112 du Bill 50. 
d) en matière de plainte pour violation de l'article 14, tout intéressé;

e) en matière de toute autre décision du commissaire-enquêteur, tout intéressé. (Rempl. Bill 50, a. 38.)

107. La partie qui désire en appeler d'une décision d'un commissaire-enquêteur doit en demander la permission à un juge désigné pour présider les audiences du tribunal, par requête signifiée aux autres parties intéressées et produite au greffe du tribunal dans les dix jours de la décision du commissaire-enquêteur. L'appelant doit également signifier cette requête au commissaire-enquêteur qui doit transmettre immédiatement le dossier de l'enquête au tribunal et en transmettre des copies aux parties.

Cette requête doit énoncer les raisons pour lesquelles l'appel est demandé et être accompagnée d'un avis du lieu, de l'heure et de la date de sa présentation, qui ne doit pas être postérieure au troisième jour qui suit le délai de dix jours spécifiés au premier alinéa.

Le juge doit rendre sa décision sur cette requête dans les cinq jours suivants.

Si le juge qui a entendu la requête devient soudainement incapable par suite d'un événement imprévu de rendre sa décision dans le délai imparti de cinq jours, le juge en chef ou, s'il est incapable d'agir, son remplaçant en désigne immédiatement un autre pour entendre sans retard et décider la requête dans le même délai.

(Rempl. Bill 50, a. 38.)

108. Le juge doit entendre l'appel dans les quinze jours de la permission d'appeler et rendre un jugement définitif dans les quinze jours de la fin de l'audition. Il doit en transmettre sans délai une copie conforme à chaque partie intéressée et au commissaire-enquêteur en chef. L'original est conservé dans un greffe facilement accessible au public.

(Rempl. Bill 50, a. 38.)

109. Une copie de toute décision du tribunal accordant une accréditation doit être transmise sans délai au ministre.

(Rempl. Bill 50, a. 38.)

110. Le juge en chef peut faire des règlements pour la régie interne du tribunal. (Rempl. Bill 50, a. 38.)

111. Le lieutenant-gouverneur en conseil, sur la recommandation du ministre et après consultation du Conseil consultatif du travail et de la main-d'oeuvre, peut faire tout règlement qu'il juge approprié pour donner effet aux dispositions $d u$ présent code.

Il peut de la même façon et en particulier établir les conditions auxquelles un salarié peut être reconnu membre d'une association, édicter les règlements applicables à la conduite de la procédure et à l'instruction des instances devant le tribunal et établir une procédure d'accréditation appropriée au caractère temporaire et saisonnier des exploitations forestières.

(Rempl. Bill 50, a. 38.) 
112. Nonobstant l'article 21 , le commissaire-enquêteur en chef, les commissaires-enquêteurs et les enquêteurs sont, jusqu'au ler juillet 1970, nommés et rémunérés d'après les effectifs, normes et barèmes, établis par règlement du ministre du travail et de la main-d'oeuvre approuvé par le lieutenant-gouverneur en conseil; s'ils sont encore en fonction à cette date, la Loi de la fonction publique leur devient alors applicable sans autres formalités; jusqu'au ler juillet 1970, le personnel qui n'est pas régi par une convention collective de travail ne peut être destitué que conformément à l'article 61 de la Loi de la fonction publique.

(Rempl. Bill 50, a. 38.)

\section{Chapitre VII}

\section{DES RECOURS ET APPELS}

121. Nulle action en vertu de l'article 33 du Code de procédure civile, ni aucun recours extraordinaire au sens de ce code, ni aucune injonction ne peuvent être exercés contre un conseil d'arbitrage, un tribunal d'arbitrage, un arbitre des griefs, un enquêteur, un commissaire-enquêteur ou le tribunal, en raison d'actes, de procédures ou de décisions se rapportant à l'exercice de leurs fonctions.

(Rempl. Bill 50, a. 39.)

122. Deux juges de la Cour du banc de la reine peuvent sur requête annuler sommairement tout bref et toute ordonnance ou injonction délivrés ou accordés à l'encontre de l'article précédent.

\section{Chapitre VIII \\ PENALITÉ}

123. Tout employeur qui, ayant reçu l'avis prescrit, fait défaut de reconnaître comme représentants de salariés à son emploi les représentants d'une association de salariés accréditée ou de négocier de bonne foi avec eux une convention collective de travail, commet une infraction et est passible d'une amende de cent à mille dollars pour chaque jour ou fraction de jour que dure l'infraction.

124. Quiconque déclare ou provoque une grève ou un lock-out contrairement aux dispositions du présent code, ou y participe, est passible, s'il s'agit d'un employeur, d'une association ou d'un officier ou représentant d'une association, d'une amende de cent à mille dollars pour chaque jour ou partie de jour pendant lequel cette grève ou ce lock-out existe et dans tous autres cas, d'une amende de dix à cinquante dollars pour chaque tel jour ou partie de jour.

125. Quiconque enfreint une disposition des articles 11,12 ou 13, commet une infraction et est passible d'une amende de cent à mille dollars pour chaque jour ou fraction de jour que dure l'infraction.

126. Quiconque fait défaut de se conformer à une obligation ou à une prohibition imposée par le présent code, ou par un règlement du lieutenant-gouverneur en conseil, ou par un règlement ou une décision d'un enquêteur, d'un commissaireenquêteur du tribunal ou d'un de ses juges, commet une infraction et est passible, à moins qu'une autre peine ne soit applicable, d'une amende de vingt-cing à cent dollars et de cent à mille dollars pour chaque récidive dans les deux ans.

(Am. Bill. 50, a. 40.) 
127. Abrogé, Bill 50, a. 41.

128. Est partie à toute infraction et passible de la peine prévue au même titre qu'une personne qui la commet toute personne qui aide à la commettre ou conseille de la commettre, et dans le cas où l'infraction est commise par une corporation ou par une association, est coupable de l'infraction tout directeur, tout administrateur, gérant ou officier qui, de quelque manière, approuve l'acte qui constitue l'infraction ou $\mathrm{y}$ acquiesce.

129. Si plusieurs personnes forment l'intention commune de commettre une infraction, chacune d'elles est coupable de chaque infraction commise par l'une d'elles dans la poursuite de la commune intention.

130. Les peines prévues par la présente loi sont imposées sur poursuite sommaire, suivant la Loi des convictions sommaires de Québec.

La deuxième partie de la dite loi s'applique aux dites poursuites.

131. Toute poursuite pénale en vertu du présent code peut être intentée par le commissaire-enquêteur en chef ou une partie intéressée.

(Rempl. Bill 50, a. 42.)

132. S'il est prouvé au tribunal qu'une association a participé à une infraction aux dispositions de l'article 11 , il peut, sans préjudice de toute autre peine, prononcer la dissolution de cette association après lui avoir donné l'occasion d'être entendue et de faire toute preuve tendant à se disculper.

S'il s'agit d'un syndicat professionnel, une copie authentique de la décision est transmise au secrétaire de la province qui en donne avis dans la Gazette officielle du Québec.

(Rempl. Bill 50, a. 43.)

\section{Chapitre IX}

DE LA PROCÉDURE

133. Tout employeur, toute association peut se faire représenter pour les fins du présent code par des représentants dûment mandatés.

134. Aucune procédure faite en vertu du présent code ne doit être considérée comme nulle ou rejetée pour vice de forme ou irrégularité de procédure.

135. Aucune preuve n'est permise pour établir qu'une enquête ou poursuite prévue par le présent code a été intentée à la suite d'une plainte d'un dénonciateur ou pour découvrir l'identité de ce dernier.

DISPOSITIONS TRANSITOIRES

136. Abrogé, Bill 50, a. 44.

137. Les certificats de reconnaissance syndicale actuellement en vigueur restent valides et équivalent à une accréditation en vertu du présent code.

Pour les instituteurs au sens de la Loi de l'instruction publique (chap. 235), il en est de même des certificats de reconnaissance syndicale en vigueur le 17 juin 1965. (13-14 Elizabeth II, 1965, chap. 50, a. 6) 
138. Les conventions collectives et sentences arbitrales actuellement en vigueur ont la portée et les effets de conventions collectives conclues ou de sentences arbitrales rendues suivant le présent code auquel elles deviennent assujetties.

Pour les instituteurs au sens de la Loi de l'instruction publique (chap. 235), il en est de même des conventions collectives et sentences arbitrales en vigueur le 17 juin 1965. (13-14 Elizabeth II, 1965, chap. 50, a. 7)

139. Les conseils d'arbitrage formés en vertu de la Loi des différends ouvriers de Québec, de la Loi des relations ouvrières ou de la Loi des différends entre les services publics et leurs salariés continuent d'exister comme s'ils avaient été formés en vertu du présent code.

140. Les conseils d'arbitrage constitués en vertu de la Loi concernant les corporations municipales et scolaires et leurs employés (13 George VI, chapitre 26) continuent d'exister aux seules fins de terminer les instances dont ils sont saisis lors de l'entrée en vigueur du présent code pour les corporations municipales, le 17 juin 1965, pour les corporations scolaires. (13-14 Elizabeth II, 1965, chap. 50, a. 8)

141. (13-14 Elizabeth II, 1965, chap. 50, a. 9)

142. Les membres de la Sûreté provinciale du Québec ne peuvent être membres d'une association de salariés ou d'une association affiliée à une telle association et toute grève leur est interdite.

143. (13-14 Elizabeth II, 1965, chap. 14, a. 77)

144. Le présent code entrera en vigueur le ler septembre 1964.

\section{Dispositions transitoires \\ Bill 50}

45. Toute affaire pendante devant la Commission des relations du travail du Québec lors de l'entrée en vigueur de la présente loi est déférée, pour décision, à un enquêteur ou à un commissaire-enquêteur agissant sous l'autorité du Code du travail selon les juridictions qui leur sont attribuées respectivement et en observant la procédure et les formalités prescrites en la matière par ledit code.

46. Est nulle et sans effet toute convention collective conclue par une association reconnue et qui a été déposée selon l'article 60 du Code du travail.

47. La Loi de la fonction publique est modifiée en remplaçant les mots «La Commission des relations de travail du Québec», partout où ils se rencontrent, par les mots «le commissaire-enquêteur en chef désigné dans le Code du travail».

48. Les personnes qui sont membres de la Commission des relations de travail du Québec, le 1er juin 1969, sont réputées, spécialement aux fins du troisième alinéa de l'article 47 du Régime de retraite des fonctionnaires, avoir été en fonctions en cette qualité pendant au moins cinq ans.

Ces personnes deviennent, à compter du (insérer ici la date de l'entrée en vigueur $d u$ bill 50), aux mêmes traitements et conditions, des fonctionnaires du ministère du travail et de la main-d'oeuvre.

49. La présente loi entrera en vigueur à la date ou aux dates fixées par une ou plusieurs proclamations, du lieutenant-gouverneur en conseil. 